\title{
$\Pi_{2}^{1}$ SETS AND $\Pi_{2}^{1}$ SINGLETONS
}

\section{LEO HARRINGTON}

ABSTRACT. The following are equivalent:

(a) every real is constructible;

(b) every nonempty $\Pi_{2}^{1}$ set of reals contains a $\Pi_{2}^{1}$ singleton.

(Implication (a) $\Rightarrow(\mathrm{b})$ is due solely to H. Friedman.)

We refer the reader to [6] and [9] for the pertinent notation and definitions.

One of the most fruitful activities in effective descriptive set theory has been the computing of bases for various levels of the projective hierarchy. (See [4] for a summary and for the most recent developments.) The central result is undoubtably the Kondo-Addison theorem: A nonempty $\Pi_{1}^{1}$ (a) set of reals contains a $\Pi_{1}^{1}(a)$ singleton, uniformly in a (for reals $a$ ). This establishes uniformization for $\Pi_{1}^{1}$. Uniformization for $\Sigma_{2}^{1}$ is an immediate consequence of this. Since uniformization implies reduction, uniformization cannot hold on both sides of a level of the projective hierarchy (see [2] for details). Hence uniformization for $\Pi_{2}^{1}$ fails.

But what if we drop the uniformity from uniformization? As H. Friedman has shown, if every real is constructible, then every nonempty $\Pi_{2}^{1}$ set of reals contains a $\Pi_{2}^{1}$ singleton. (Friedman has actually established something more general-see Theorem 1 below.) $)^{1}$ Our main result ${ }^{2}$ is the converse of this, namely: If there is a nonconstructible real then there is a nonempty $\Pi_{2}^{1}$ set of reals which contains no $\Pi_{2}^{1}$ singleton.

Let $R=$ the set of reals, where a real is a subset of $\omega=$ the integers. Let $a, b, c, \cdots$ denote reals. By the usual standard devices, code a pair of reals or a countable set of reals by one single real.

Definition [10]. A well ordering $<$ of $R$ is strongly $\Delta_{n}^{1}$ if: $<$ is $\Delta_{n}^{1}$, $<$ has order type $\kappa_{1}$, and $\{a \in R: a$ codes an initial segment of $<\}$ is $\Sigma_{n}^{1}$.

The main feature of a strongly $\Delta_{n}^{1}$ well ordering, $<$, is that quantification over $\{b: b<c\}$ is transformed (in a $\sum_{n}^{n}(c)$ way uniformly in $c$ ) into number quantification (by using a real which codes $\{b: b<c\}$ ). Thus such quantification will not increase a hierarchy calculation. Notice in addition that each

Received by the editors July 1, 1974.

AMS (MOS) subject classifications (1970). Primary 02K 30 . reals.

Key words and phrases. Strongly $\Delta_{n}^{1}$ well ordering, $\Pi_{2}^{1}$ singletons, constructible

1 We wish to thank H. Friedman for consenting to the inclusion of the proof of his result in this paper.

2 We would also like to commend Harvey Friedman for persistently affirming his belief in this result-despite our equally persistent assurance otherwise. 
real $<c$ is $\Delta_{n}^{1}(c)$ (since the first (wrt $<$ ) real which codes $\{b: b<c\}$ is $\left.\Delta_{n}^{1}(c)\right)$.

The orem 1 (H. Friedman). If there is a strongly $\Delta_{n}^{1}$ well ordering of $R$, then every nonempty $\Pi_{n}^{1}$ set of reals contains a $\Pi_{n}^{1}$ singleton.

Proof. Let $<$ be a strongly $\Delta_{n}^{1}$ well ordering of $R$. We recall a convenient definition in this context. (The following definition is slightly nonstandard. It can be made standard by replacing the real $b$ with the ordinal: the order type of $\{c: c<b\}_{.}$)

Definition. $b \in R$ is called stable if: for all $d<b$ and all $\Pi_{n-1}^{1}$ formulas $\theta(x, y),(\exists x) \theta(x, d) \Rightarrow(\exists x<b) \theta(x, d)$.

For a real $c, \delta_{n}^{1}(c)=$ the first $($ wrt $<$ ) stable real $>c$.

Notice that for reals $c, a, a$ is $\Delta_{n}^{1}(c)$ iff $a<\delta_{n}^{1}(c)$. [Proof. (This is folklore.) If $a$ is $\Delta_{n}^{1}(c)$ then there is a $\Pi_{n-1}^{1}$ formula $\theta(w, x, y)$ such that $(\exists w) \theta(w, x, c) \Leftrightarrow x=a$. Thus for some $w,\langle w, a\rangle\left\langle\delta_{n}^{1}(c)\right.$ and hence $a<$ $\delta_{n}^{1}(c)$. For the other direction, let $b=$ the first (wrt $<$ ) real not $\Delta_{n}^{1}(c)$. Given $d<b$ and given a $\Pi_{n-1}^{1}$ formula $\theta(x, y)$, if $(\exists x) \theta(x, d)$ then the first (wrt <) such $x$ is $\Delta_{n}^{1}(d)$ and hence $x<b$. Thus $b$ is stable, so $b=\delta_{n}^{1}(c)$. Hence $a<\delta_{n}^{1}(c)=b \Rightarrow a$ is $\Delta_{n}^{1}(c)$.]

Now let $Y$ be a nonempty $\Pi_{n}^{1}$ set of reals. Let $a=$ the first (wrt $<$ ) real in $Y$. We claim that $\{a\}$ is $\Pi_{n}^{1}$.

For some $\Sigma_{n-1}^{1}$ formula $\psi(x, y)$ we have: $y \in Y$ iff $\forall x \psi(x, y)$. Let $W=$ $\{b:(\forall c<b)(\exists d<b) \neg \psi(d, c)\} . W$ is $\Delta_{n}^{1}$. Notice that if $b$ is stable and if $b \leq a$, then $b \in W$. Also notice that $b \in W \Rightarrow b \leq a$.

Let $c=$ the first (wrt $<$ ) real which is $\geq b$ for all $b \in W$. An examination of the definition of $W$ reveals that $c \in W$. Clearly $c \leq a . c$ is uniformly $\Delta_{n}^{1}(d)$ for all $d \geq a$; that is, there is a $\Sigma_{n}^{1}$ formula $\phi(z, x)$ such that: for all $d \geq a, \phi(z, d) \Leftrightarrow z=c$. $\phi(z, x)$ simply says: $z \in W$ and $(\forall w \leq x)$ $(w \in W \Rightarrow w \leq z)$.

Now, $\delta_{n}^{1}(c)>c$, and thus $\delta_{n}^{1}(c) \notin W$. But $\delta_{n}^{1}(c)$ is stable. Hence $a<$ $\delta_{n}^{1}(c)$, and so $a$ is $\Delta_{n}^{1}(c)$. Thus there is a $\sum_{n}^{1}$ formula $\theta(y, z)$ such that $\theta(y, c) \Leftrightarrow y=a$.

But now we have: $x=a$ iff $x \in Y$ and $\forall z \forall y[(\phi(z, x)$ and $\theta(y, z)) \Rightarrow$ $y=x]$, which is $\Pi_{n}^{1}$.

We should point out that the $\Pi_{n}^{1}$ singletons produced by the above proof are of a rather different species than $\Pi_{1}^{1}$ singletons. It is well known that a real of the same $\Delta_{1}^{1}$ degree as a $\Pi_{1}^{1}$ singleton is also a $\Pi_{1}^{1}$ singleton. The corresponding fact is not true for $\Pi_{n}^{1}$ singletons under the hypothesis of the above theorem. For example, let $a$ be the first (wrt $<$ ) real such that: for each $\Pi_{n}^{1}$ formula $\phi(x)$, if $\phi(a)$ then $\exists x\left(\phi\left(x_{1}\right)\right.$ and $x$ is strictly $\Delta_{n}^{1}$ in $\left.a\right)$, a is not a $\Pi_{n}^{1}$ singleton, but it can be verified that $a$ has the same $\Delta_{n}^{1}$ degree as a $\Pi_{n}^{1}$ singleton. 
The constructible reals are known (see [1]) to have a strongly $\Delta_{2}^{1}$ well ordering. Thus

Corollary. If every real is constructible, then every nonempty $\Pi_{n}^{1}, n \geq 2$, set of reals contains a $\Pi_{n}^{1}$ singleton.

Theorem 2。 There is a $\Pi_{2}^{1}$ predicate $\phi(x)$ such that $\mathrm{ZF} \vdash \exists x \phi(x)$, and $\mathrm{ZF}+$ "there is a nonconstructible real" $\vdash \forall x\left(\phi(x) \Rightarrow x\right.$ is not a $\Pi_{2}^{1}$ singleton).

Proof. Intuitively, $\phi(x)$ will assert (among other things) that $x$ is a member of every reasonably large $\Sigma_{2}^{1}$ set of reals, thus making it impossible for $R \sim\{x\}$ to be $\Sigma_{2}^{1}$ (unless of course every real is constructible, in which case $R \sim\{x\}$ just is not large enough). We now express this assertion in a $\Pi_{2}^{1}$ way.

A real is a subset of $\omega$, and hence may be viewed as an infinite sequence of 0 's and 1's. Let $B=2^{<\omega}=$ the full binary tree $=$ the set of all finite sequences of 0 's and 1's. For $s, t \in B, s \subseteq t$ if $t$ extends $s ; s \mid t$ if $s$ and $t$ have no common extension; $l(s)=$ the length of $s$. A tree $T$ is a subset of $B$ such that: $t \in T, s \subseteq t \Rightarrow s \in T ; s \in T \Rightarrow(\exists t, u \in T)(s \subseteq t, u$ and $t \mid u)$. A branch through $T$ is a real $b$ such that every initial segment of $b$ is in $T$; $[T]$ is the set of all branches through $T$. For $s \in T$, let $T_{s}=\{t \in T: s \subseteq t$ or $t \subseteq s\}$.

We will use the following enumeration of $\Sigma_{2}^{1}$ sets: let $\phi(e, x)$ be a universal $\Sigma_{1}$ formula of the language of set theory; the eth $\Sigma_{2}^{1}$ set of reals, $A(e)$, is defined by, $x \in A(e)$ iff $L[x] \vDash \phi(e, x)$. (This does enumerate all $\Sigma_{2}^{1}$ sets of reals. See [8]. For an ordinal $\alpha$, let $A(e, \alpha)=\left\{x \in R: L_{\alpha}[x] \vDash\right.$ $\phi(e, x)\}$. (If $\alpha$ is countable then $A(e, \alpha)$ is Borel. This is nothing more than the usual decomposition of a $\Sigma_{2}^{1}$ set into a union of $\kappa_{1}$ Borel sets.) If $T$ is a tree, then the assertion: " $[T] \subseteq A(e, \alpha)$ " is $\Pi_{1}^{1}$ over the structure $L_{\alpha}[T]$, and hence is absolute for countable $\alpha$ 's. We will use without explicit mention the fact that similar statements are also absolute.

We will need the following result of Solovay [11] and Mansfield [5]:

The perfect set theorem. If $T$ is a tree in $L$, and if $[T] \cap A(e)$ contains a nonconstructable real, then for some tree $T^{\prime} \subseteq T$ and some $\alpha<\boldsymbol{\kappa}_{1}^{L},\left[T^{\prime}\right] \subseteq$ $A(e, \alpha)$.

We now define, for all ordinals $j \leq \lambda$ (where $\lambda$ will eventually turn out to be the ordinal $\left.\delta_{2}^{1}\right)$, an ordinal $a(j)$, a set of integers $Z(j)$, and a set of trees $X(j)$. The map $j \mapsto a(j), Z(j), X(j)$ will be $\Sigma_{1}$ over $L_{\boldsymbol{\kappa}_{1} L^{\cdot}}$ (So in particular each tree in $X(j)$ is constructible.) In addition, the following properties will hold.

For $j \leq \lambda$ and for $T \in X(j)$ :

(i) For all $i<j, \alpha(i)<\alpha(j)$ and $Z(i) \subset Z(j)$.

(ii) $s \in T \Rightarrow T_{s} \in X(j)$; for all $i<j$ and all $S \in X(i),\left(\exists S^{\prime} \in X(j)\right)\left(S^{\prime} \subseteq S\right)$. 
(iii) For all $i<j,[T] \subseteq \bigcup\{[S]: S \in X(i)\}$.

(iv) $e \in Z(j) \Rightarrow[T] \subseteq A(e, \alpha(j))$.

The following should be reminiscent of Jensen's construction in [3].

Case 1. $j=0$. Let $\alpha(0)=0, Z(0)=\varnothing, X(0)=\left\{B_{s}: s \in B\right\}$.

Case 2. $j$ a limit ordinal. We are given $\alpha(i), Z(i), X(i)$ for $i<j$. We claim that for each $k<j$ and each $U \in X(k)$, there is a tree $T \subseteq U$ such that $T$ satisfies (iii) above. [ $T$ is found by fusion, as in [7] or [3, p. 124]. Let $k=i_{0}<i_{1}<\cdots$ be such that $U_{n} i_{n}=j$. Using (ii) above, find for each $t \in B$ a tree $T^{t}$ such that:

$$
\begin{aligned}
& T^{\varnothing}=U, l(t)=n \Rightarrow T^{t} \in X\left(i_{n}\right), \\
& u \subseteq t \Rightarrow T^{t} \subseteq T^{u}, \\
& u \mid t \Rightarrow\left[T^{u}\right] \cap\left[T^{t}\right]=\varnothing, \text { and } \\
& l(t)=n \Rightarrow T^{t}=\left(T^{t}\right)_{s} \text { where } l(s)=n .
\end{aligned}
$$

Let $T=\bigcap_{n} \bigcup\left\{T^{t}: t \in B\right.$ and $\left.l(t)=n\right\}$. It can be checked that $T$ is a tree. Clearly $T \subseteq T^{\varnothing}=U$. For each $n,[T] \subseteq \bigcup\left\{\left[T^{t}\right]: l(t)=n\right\}$. Thus $[T] \subseteq \bigcup\{[S]$ :

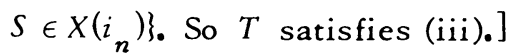

For each $k<j$ and each $U \in X(k)$, let $U^{\prime}=$ the first (first in the sense of $L)$ tree $T \subseteq U$ such that $T$ satisfies (iii).

Let $X(j)=\left\{U_{s}^{\prime}: U \in X(k)\right.$ for some $k<j$, and $\left.s \in U^{\prime}\right\}$. So (ii) and (iii) are satisfied. Let $a(j)=U_{i<j} \alpha(i)$, and let $Z(j)=U_{i<j} Z(i)$. So (i) holds. Since (iii) holds, so does (iv).

Case 3. $j=i+1$. (This is the only interesting case.) We are given $a(i)$, $Z(i), X(i)$. Let $\alpha(j)=$ the first $\alpha>\alpha(i), \alpha<\boldsymbol{x}_{1}^{L}$, for which there is $e \notin Z(i)$ such that $(\forall T \in X(i))$ ( $\exists$ a tree $\left.T^{\prime} \subseteq T, T^{\prime} \in L_{\alpha}\right)\left(\left[T^{\prime}\right] \subseteq A(e, \alpha)\right)$. [If there is no such $\alpha$, then we let $\lambda=i$, and we leave $\alpha(j), Z(j), X(j)$ undefined.] Let $e_{0}$ be the first such $e$. Let $Z(j)=Z(i) \cup\left\{e_{0}\right\}$. For each $T \in X(i)$, let $T^{\prime}=$ the first (first in the sense of $L$ ) tree such that $T^{\prime} \subseteq T$ and $\left[T^{\prime}\right] \subseteq A\left(e_{0}, \alpha(j)\right)$. Let $X(j)=\left\{T_{s}^{\prime}: T \in X(i)\right.$ and $\left.s \in T^{\prime}\right\}$. Clearly (i) through (iv) are satisfied.

This completes the definition of $\alpha(j), Z(j), X(j), j \leq \lambda$.

Remarks. (1). It is easy to see that the above construction continues for at least $\delta_{2}^{1}$ steps (i.e., if $i<\delta_{2}^{1}$ then $\alpha(i+1)$ is defined). But $Z(\lambda)$ is clearly a $\Sigma_{2}^{1}$ set of integers, and hence $\lambda=\delta_{2}^{1}$.

(2). If $e \notin Z(\lambda)$, then (since $\alpha(\lambda+1)$ is undefined) there is $T \in X(\lambda)$ such that for all trees $T^{\prime} \subseteq T$ and all $\alpha<\boldsymbol{\kappa}_{1}^{L},\left[T^{\prime}\right] \not \subset A(e, \alpha)$.

We can now produce the promised $\Pi_{2}^{1}$ predicate $\phi$. For $x \in R$, let

$$
\phi(x) \equiv(\forall j \leq \lambda)(\forall e \in Z(j))(x \in A(e, \alpha(j))) .
$$

Since the map $j \mapsto \alpha(j), Z(j)$ is $\Sigma_{1}$ over $L_{\aleph_{1}}, \phi$ is $\Pi_{2}^{1}$.

By (iii) and (iv), every branch through a member of $X(\lambda)$ is a solution of $\phi$. Thus $\mathrm{ZF} \vdash j x \phi(x)$. (Notice that $\phi$ is actually $\Delta_{1}^{1}$ in any real which enumerates $\lambda_{0}$ ) 
Now suppose we are in a model of $\mathrm{ZF}$, and we have a real $b$ such that $\phi(b)$ and such that $\{b\}$ is $\Pi_{2}^{1}$. For some $e,\{b\}=R \sim A(e)$. Since $b \notin A(e)$, $e \notin Z(\lambda)$. Thus there is $T \in Z(\lambda)$ as described in Remark (2). But then by the perfect set theorem mentioned earlier, every nonconstructible branch through $T$ is in $R \sim A(e)$. Thus [T] has at most one nonconstructible member. Since $T \in L$, this means that there are no nonconstructible reals.

We should mention in closing that Theorem 2 seems optimal. By [3] there are models of ZFC with nonconstuctible reals in which every nonempty $\Pi_{2}^{1}$ set contains a $\Delta_{3}^{1}$ real.

\section{REFERENCES}

1. J. W. Addison, Some consequences of the axiom of constructibility, Fund. Math. 46 (1959), 337-357. MR 23 \#A1523.

2. - Separation principles in the hierachies of classical and effective descriptive set theory, Fund. Math. 46 (1959), 123-135. MR 24 \#A1209.

3. R. B. Jensen, Definable sets of minimal degree, Mathematical Logic and Foundations of Set Theory (Proc. Internat. Colloq., Jerusalem, 1968), North-Holland, Amsterdam, 1970, pp. 122-128. MR 46 \#5130.

4. A. S. Kechris, Measure and category in effective descriptive set theory, Ann. of Math. Logic 5 (1973), 337-384.

5. R. Mansfield, Perfect subsets of definable sets of reals, Pacific J. Math. 35 (1970), 451-457. MR $43 \# 6100$.

6. H. Rogers, Jr., Theory of recursive functions and effective computability, McGraw-Hill, New York, 1967. MR 37 \#61.

7. G. E. Sacks, Forcing with perfect closed sets, Proc. Sympos. Pure Math., vol. 13, part 1, Amer. Math. Soc., Providence, R. I., 1971, pp. 331-355. MR 43 \#1827.

8. J.R. Shoenfield, The problem of predicativity, Essays on the foundations of math., Magnes Press, Hebrew Univ., Jerusalem, 1961, pp. 132-139. MR 29 \#2177. $\# 1224$.

9. - Mathematical logic, Addison-Wesley, Reading, Mass., 1967. MR 37

10. J. H. Silver, Measurable cardinals and $\Delta_{3}^{1}$ well-orderings, Ann. of Math. (2) 94 (1971), 414-446. MR $45 \# 8517$.

11. R. M. Solovay, On the cardinality of $\Sigma_{2}^{1}$ sets, Foundations of Mathematics (Sympos. Commemorating Kurt Gödel, Columbus, Ohio, 1966 ), Springer, New York, 1969, pp. 58-73. MR $43 \# 3115$.

DEPARTMENT OF MATHEMATICS, STATE UNIVERSITY OF NEW YORK AT BUFFALO, AMHERST, NEW YORK 14226

Current address: Department of Mathematics, University of California, Berkeley, California 94720 